\title{
Direct Observation of Hydrodynamic Rotation-Translation Coupling between Two Colloidal Spheres
}

\author{
S. Martin, ${ }^{*}$ M. Reichert, H. Stark, ${ }^{\dagger}$ and T. Gisler ${ }^{\ddagger}$ \\ Universität Konstanz, Fachbereich Physik, 78457 Konstanz, Germany
}

(Received 13 July 2006; published 15 December 2006)

\begin{abstract}
By combining optical tweezers with polarization microscopy, the hydrodynamic coupling between position and orientation fluctuations in a pair of colloidal spheres has been measured. Imaging of birefringent particles under crossed polarizers allows for the simultaneous determination of the positions and orientations of both particles. The temporal cross-correlation function between random displacements of one particle and orientation fluctuations of its neighbor allows for the quantification of the hydrodynamic rotation-translation coupling between the spheres. Our results are in good agreement with predictions for the hydrodynamic mobility tensors calculated in the creeping-flow limit of the NavierStokes equation.
\end{abstract}

PACS numbers: 47.57.J-, 05.40.Jc, 45.20.dc, 47.15.G-

The presence of long-range, solvent-mediated hydrodynamic interactions (HI) in complex fluids and biological systems leads to fascinating phenomena which have no counterpart in the realm of simple liquids. We mention fractional density dependence of sedimentation velocities in suspensions of charged particles [1] and long-range correlations in sedimenting hard spheres [2], synchronization of beating cilia or rotating flagella driven by biological motors [3-5], or pattern formation in confined arrays of spermatozoa [6]. HI arise from the velocity field in the solvent excited by the (random or directed) motion of a particle. This velocity field propagates over the interparticle distance $R$ within the vorticity time $\tau_{\nu} \sim R^{2} / \nu$, where $\nu$ is the kinematic viscosity of the solvent. A neighboring particle thus experiences a drag force proportional to its velocity relative to the velocity induced by the first particle. Since particles diffuse over only a minute fraction of the interparticle distance within $\tau_{\nu}$, hydrodynamic coupling can be considered instantaneous on time scales much larger than $\tau_{\nu}$.

As the flow disturbance induced by a position fluctuation of one particle is inhomogeneous, the resulting viscous stress at the surface of its neighbor should also result in a drag torque. Indeed, a detailed analysis in the creepingflow limit of the Navier-Stokes equation shows that hydrodynamic interactions lead to coupling between orientation of one particle and the transverse displacement of its neighbor in the direction perpendicular to the interparticle separation, while orientations and longitudinal displacements (parallel to the interparticle separation) are decoupled due to symmetry [7,8]. In contrast to the $1 / R$ dependence of the translation-translation coupling $[7,8]$ which was recently directly demonstrated in experiments $[9,10]$, the mobility coefficient describing rotationtranslation coupling is expected to decay faster, namely, as $1 / R^{2}[7,8]$. Measurements of the orientational relaxation in suspensions of spherical colloidal particles by depolar- ized quasielastic light scattering have indicated the presence of hydrodynamic coupling between translation and rotation [11]. However, the detailed comparison of theoretical predictions with experimental data is complicated by the fact that measurable quantities sensitive to rotationtranslation coupling, such as the rotational diffusion coefficient, are related to the mobility coefficients by configuration-space averages requiring a detailed knowledge of the microstructure of the suspension [12].

In this Letter we directly measure hydrodynamic rotation-translation coupling by simultaneously determining position and orientation fluctuations of a pair of spherical colloidal particles each held in a calibrated optical tweezer at fixed average distance $R$. For our experiments we used nematic liquid crystal droplets of reactive monomer RM257 with diameters $3 \mu \mathrm{m} \leq 2 a \leq 4 \mu \mathrm{m}$, whose bipolar nematic order had been locked by polymerization $[13,14]$. Samples were prepared by making a dilute mixture of particles (volume fraction $<10^{-2}$ ) suspended in an aqueous sodium lauryl sulfate solution $(10 \mathrm{mM})$ in order to stabilize against aggregation. Some of this solution was then placed in a $170 \mu \mathrm{m}$ thick sample cell which was hermetically sealed with UV curing glue. Particles were trapped at a distance of about $20 \mu \mathrm{m}$ above the bottom cell surface. Particle diameters $2 a$ were estimated to within approximately $0.2 \mu \mathrm{m}$ by measuring a focused image of the particles. For all measurements we chose particles with matched diameters, and the temperature was kept at $T=$ $288 \pm 0.5 \mathrm{~K}$ with a calibrated Peltier cooling stage.

In order to measure position and orientation fluctuations of the particles simultaneously via direct imaging, we use a dual-beam optical tweezer setup designed similar to that of Fällman and Axner [15]. A linearly polarized laser beam with wavelength $\lambda_{0}=532 \mathrm{~nm}$ is split by a nonpolarizing beam splitter and directed towards two separate gimbalmounted mirrors used to steer the two traps independently. The two beams are then passed through polarizing ele- 
ments used to control the polarization state of the traps and recombined onto the back focal plane of an oil immersion microscope objective (Zeiss Plan Neo-Apochromat $100 \times$, numerical aperture of 1.4). This objective is used for both trapping and imaging the particles onto an 8-bit CCD camera (Lavision HSS-1). The strength of each trap is about $8 \mathrm{~mW}$, in order to strike a balance between the magnitudes of position and orientation fluctuations. However, at these trapping strengths we typically find the particle escaping the trap after about seven to ten minutes. Thus the optimum time for each measurement, taking image resolution and computer processing time into account, is approximately $270 \mathrm{~s}$ (32 678 images at 125 frames per second with an exposure time of $2 \mathrm{~ms}$ ). The same two particles are typically used to perform 8 repeat measurements, amounting to a total experiment duration of $2000 \mathrm{~s}$.

Longitudinal and transverse particle displacements $\Delta x_{1,2}(t)$ and $\Delta y_{1,2}(t)$ (with respect to the mean interparticle separation vector $\boldsymbol{R}$, see Fig. 1) were determined with a center-finding algorithm [16]. Positions were measured with subpixel accuracy to an uncertainty of $\pm 10 \mathrm{~nm}$. The distributions $p_{i}\left(\Delta x_{i}, \Delta y_{i}\right)$ of the displacements $\Delta x_{i}$ and $\Delta y_{i}$ were found to be isotropic and Gaussian for both foci. Mean-square displacements $\left\langle\Delta x_{1,2}^{2}\right\rangle$ and $\left\langle\Delta y_{1,2}^{2}\right\rangle$ were measured by fitting a Gaussian to the distributions. Rootmean-square thermal displacements were around $100 \mathrm{~nm}$ for most experiments. The average spring constants $\kappa_{1,2}$ of the optical gradient potential were determined from the equipartition theorem $\kappa_{i}=k_{\mathrm{B}} T /\left\langle\Delta x_{i}^{2}\right\rangle=k_{\mathrm{B}} T /\left\langle\Delta y_{i}^{2}\right\rangle$.

The autocorrelation function of the transverse position fluctuations, $C_{y_{i} y_{i}}(\tau)=\left\langle\Delta y_{i}(t) \Delta y_{i}(t+\tau)\right\rangle /\left\langle\Delta y_{i}^{2}\right\rangle$, are found to decay approximately exponentially with decay times of about $0.2 \mathrm{~s}$, consistent with the estimate $\tau_{i}^{(\mathrm{t})}=\zeta^{(\mathrm{t})} / \kappa_{i}=$ $0.17 \mathrm{~s}$ for noninteracting particles, using the measured trap stiffnesses $\kappa_{i}=(2.6 \pm 0.1) \times 10^{-7} \mathrm{Nm}^{-1}$, the translational friction factor $\zeta^{(\mathrm{t})}=6 \pi \eta a$, and the viscosity of water $\eta=1.14 \times 10^{-3} \mathrm{Pas}$ [17]. This shows that the influence of hydrodynamic coupling between the two spheres on the autocorrelation functions $C_{y_{i} y_{i}}(\tau)$ is small [18].

In order to measure the angular fluctuations $\Delta \theta_{1,2}(t)$ of the particles in the traps about the $z$ axis, they are viewed between crossed polarizer and analyzer. Because of the bipolar nematic structure within the particle, light transmitted through the particle is elliptically polarized, leading to a particle image varying in brightness with the angle of the particle director with the analyzer. The spectrum of the illumination source was limited to wavelengths between $610 \mathrm{~nm}$ and $690 \mathrm{~nm}$ by an infrared filter behind the lamp and a color filter before the camera in order to reduce chromatic aberration by the particles and to optimize the contrast of the detection to better than 100:1. In order to convert transmitted intensity to angle, the particles are trapped with linearly polarized tweezer beams whose common polarization vector $\boldsymbol{E}$ is oriented with an angle $\theta_{0}=$ (a)

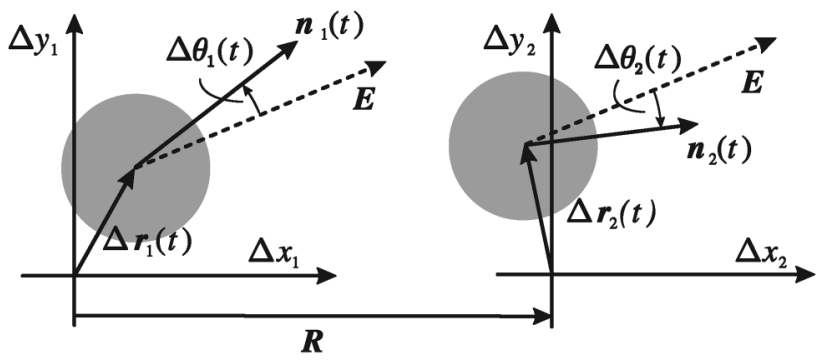

(b)

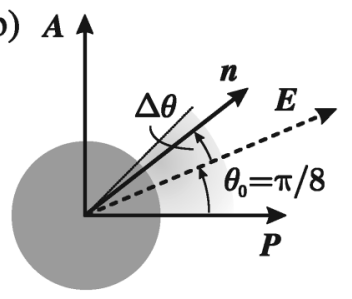

(c)

$+$

\#

FIG. 1. (a) Two particles held at fixed average separation $R$ by the two tweezer beams propagating along the $z$ axis undergo thermal position fluctuations $\Delta \boldsymbol{r}_{1,2}$ around their average positions. The longitudinal and transverse components $\Delta x_{1,2}$ and $\Delta y_{1,2}$, respectively, are defined with respect to the average separation vector $\boldsymbol{R}$. The angular fluctuations $\Delta \theta_{1,2}$ are defined by $\boldsymbol{n}_{1,2}$, the particle directors projected onto the $x y$ plane, and the tweezer polarization $\boldsymbol{E}$. (b) Restricting the angular fluctuations $\Delta \theta$ to within $\pm \pi / 8$ (light shaded area) around the tweezer polarization $\boldsymbol{E}$ allows for their direct determination from the intensities $I(\Delta \theta) \sim 1+\sin (4 \Delta \theta)$ transmitted through the analyzer $\boldsymbol{A}$. Positive fluctuations $\Delta \theta_{i}>0$ are in the counterclockwise direction. $\boldsymbol{E}$ is oriented at an angle $\theta_{0}=\pi / 8$ with the polarizer $\boldsymbol{P}$ such that the maximal and minimal transmitted intensities, $I_{\max }$ and $I_{\min }$, occur at $\Delta \theta=\pi / 8$ and at $\Delta \theta=$ $-\pi / 8$, respectively. (c) Two birefringent particles with equal diameters $2 a=3.5 \mu \mathrm{m}$ at a distance $R=6.8 \mu \mathrm{m}$ viewed under crossed polarizer and analyzer. Trapping foci are about $0.5 \mu \mathrm{m}$ in diameter.

$\pi / 8$ with the polarizer, such that the average intensity $\langle I\rangle$ is halfway between the minimal and the maximal intensities $I_{\min }$ and $I_{\max }$, respectively [see Fig. 1(b)]. $I_{\min }$ and $I_{\max }$ are determined by recording the particle brightness under continuous rotation of the particles with circularly polarized tweezer beams (see Fig. 1). Provided the linearly polarized tweezer beams are strong enough to keep the angular fluctuations $\Delta \theta$ to within $\pm \pi / 8$, they can be recovered directly from the measured intensities $I(\Delta \theta)$ transmitted through the analyzer [see Fig. 1(b)]. Angles were measured to an uncertainty of $\pm 10^{-3}$ rad.

Angular fluctuations show nearly Gaussian distributions $p_{i}\left(\Delta \theta_{i}\right)$ with very similar widths $\left\langle\Delta \theta_{i}^{2}\right\rangle^{1 / 2}$ for both traps. Using the equipartition relation $\mathrm{s}_{i}=k_{\mathrm{B}} T /\left\langle\Delta \theta_{i}^{2}\right\rangle$ we obtain values for the angular spring constant $\varsigma_{1,2}=(2.5 \pm 0.1) \times$ $10^{-19} \mathrm{Nm} \mathrm{rad}^{-2}$ for an interparticle distance $R=5.8 \mu \mathrm{m}$. Using the Boltzmann relation the angular potential $V_{i}\left(\Delta \theta_{i}\right)$ associated with the restoring torque exerted on the particles 
by the linearly polarized trap is then computed as

$$
V_{i}\left(\Delta \theta_{i}\right)=-k_{\mathrm{B}} T \ln p_{i}\left(\Delta \theta_{i}\right)
$$

As can be seen in Fig. 2, the angular potential is well described by the harmonic approximation up to energies of about $1.5 \times 10^{-20} \mathrm{~J}\left(3.7 k_{\mathrm{B}} T\right)$. However, the spring constant obtained from a parabolic fit to the data is by about $30 \%$ higher than the one obtained from the equipartition relation. The origin of this discrepancy is a slight skewness in the potential. As the angular spring constant $\varsigma_{i}=2.5 \times$ $10^{-19} \mathrm{Nm} \mathrm{rad}{ }^{-2}$ obtained from the equipartition relation fits the potential better near its minimum, we will in the following use this value.

Fluctuations in total particle brightness would also occur as a result of position fluctuations, most strongly along the propagation direction of the trapping beams. Measurements without polarizer and analyzer show intensity fluctuations which are much lower than the ones arising from angular fluctuations under crossed polarizer and analyzer, resulting in slight noise on the angles $\Delta \theta_{i}$.

Similar to the correlations between longitudinal position fluctuations $\Delta x_{i}$ mediated by hydrodynamic interactions $[9,10]$, the hydrodynamic interactions should also couple the transverse fluctuations $\Delta y_{i}[7,8,19]$. A measurement of the temporal cross-correlation function of the transverse position fluctuations,

$$
C_{y_{1} y_{2}}(\tau)=\frac{\left\langle\Delta y_{1}(t) \Delta y_{2}(t+\tau)\right\rangle}{\left\langle\Delta y_{1}^{2}\right\rangle^{1 / 2}\left\langle\Delta y_{2}^{2}\right\rangle^{1 / 2}}
$$

shows a nonmonotonic anticorrelation between $\Delta y_{1}$ and $\Delta y_{2}$ (see Fig. 3), reflecting the fact that a fluctuation $\Delta y_{2}$

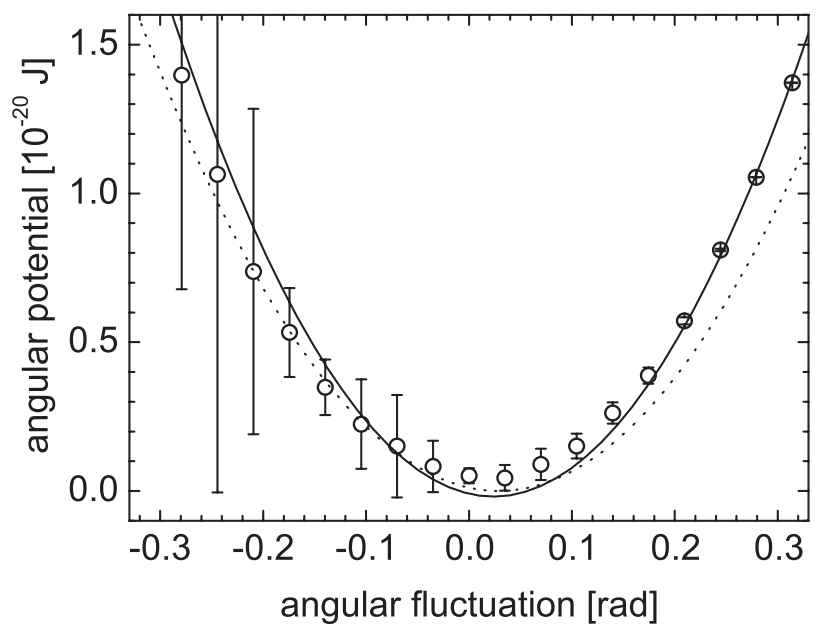

FIG. 2. Angular potential for 2 particles with diameter $2 a=$ $3.5 \mu \mathrm{m}$ at an interparticle distance $R=5.8 \mu \mathrm{m}$ computed from the histograms $p_{i}\left(\Delta \theta_{i}\right)$ using Eq. (1). Symbols are the data averaged over 2 traps. Lines: harmonic potential $V(\Delta \theta)=$ $\mathrm{s} / 2(\Delta \theta-\chi)^{2}$, with spring constant $\mathrm{s}=2.5 \times$ $10^{-19} \mathrm{Nm} \mathrm{rad}^{-2}$ and angle offset $\chi=2.9 \times 10^{-2}$ rad (dotted line) and $s=3.3 \times 10^{-19} \mathrm{Nm} \mathrm{rad}^{-2}$ and $\chi=2.9 \times 10^{-2} \mathrm{rad}$ (full line). antiparallel to $\Delta y_{1}$ relaxes more slowly than when it occurs parallel to $\Delta y_{1}$, since for parallel (collective) displacements less fluid has to be displaced than for antiparallel displacements $[9,10,19]$. The agreement with the theoretical prediction [19] is good given that there are no adjustable parameters. Although rotational terms appear in the mobility matrix, their contribution to the coupling between translational degrees of freedom is insignificant.

The inhomogeneity of the flow field induced by a transverse displacement $\Delta y_{1}$ of particle 1 should also result in an instantaneous torque acting on particle 2 , breaking the symmetry between the relaxation rates of clockwise (negative) and counterclockwise (positive) angular fluctuations $\Delta \theta_{2}$ in the orientation trap. This hydrodynamic coupling between rotation and translation is indeed revealed by the cross-correlation function

$$
C_{y_{1} \theta_{2}}(\tau)=\frac{\left\langle\Delta y_{1}(t+\tau) \Delta \theta_{2}(t)\right\rangle}{\left\langle\Delta y_{1}^{2}\right\rangle^{1 / 2}\left\langle\Delta \theta_{2}^{2}\right\rangle^{1 / 2}}
$$

of the transverse position fluctuation $\Delta y_{1}$ and the angular fluctuation $\Delta \theta_{2}$ (see Fig. 4). The data show, for short times, an increase towards a correlation peak near $\tau \approx 0.25 \mathrm{~s}$, followed by a slow decay at longer times. The calculations in the creeping-flow limit [19] show good agreement with the data. The agreement of the theoretical prediction for experimentally determined translational and rotational trap stiffnesses $\kappa_{1,2}=2.6 \times 10^{-7} \mathrm{Nm}^{-1}$ and $\mathrm{s}_{1,2}=2.5 \times$ $10^{-19} \mathrm{Nm} \mathrm{rad}^{-2}$ is very good given that there are no free parameters. The slight baseline offset in $C_{y_{1} \theta_{2}}(\tau)$ arises

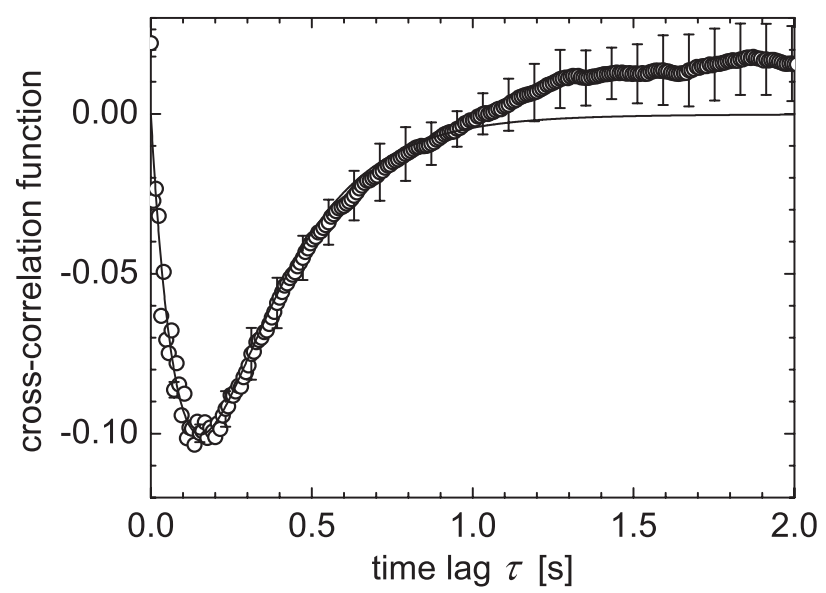

FIG. 3. Temporal cross-correlation function $C_{y_{1} y_{2}}(\tau)$ of the transverse position fluctuations $\Delta y_{1,2}(t)$ of two particles held in linearly polarized traps restraining both position and angular fluctuations, for particles with equal diameters $2 a=3.5 \mu \mathrm{m}$ held at an average interparticle separation $R=5.8 \mu \mathrm{m}$. The full line is the prediction Eq. (34) from [19] including hydrodynamic coupling, with spring constants $\kappa_{1}=\kappa_{2}=$ $2.6 \times 10^{-7} \mathrm{Nm}^{-1}$, and angular spring constants $\varsigma_{1}=\varsigma_{2}=$ $2.5 \times 10^{-19} \mathrm{Nm} \mathrm{rad}^{-2}$ measured directly from the distributions $p_{i}\left(\Delta y_{i}\right)$ and $p_{i}\left(\Delta \theta_{i}\right)$. Error bars represent the standard deviation over 8 measurements. 


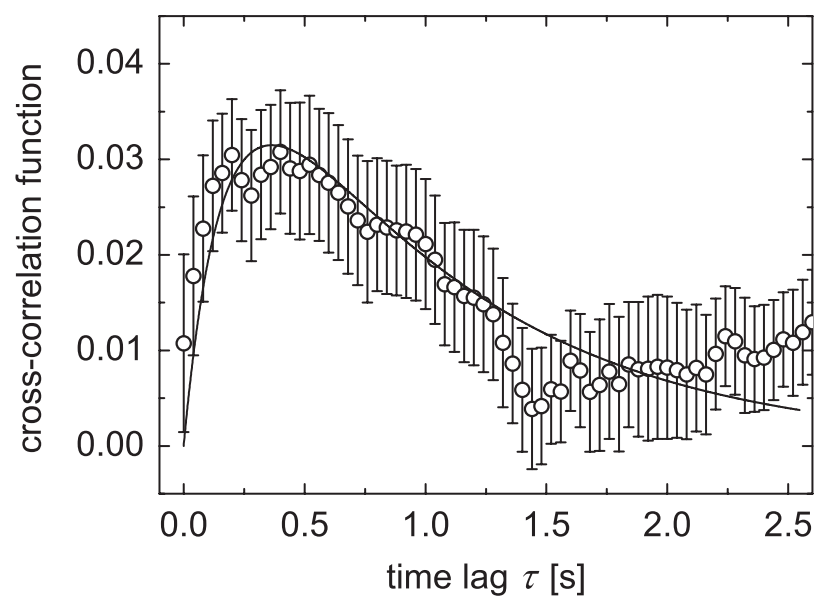

FIG. 4. Cross-correlation function $C_{y_{1} \theta_{2}}(\tau)$ of the position and angular fluctuations $\Delta y_{1}$ and $\Delta \theta_{2}$ at interparticle distance $R=$ $5.8 \mu \mathrm{m}$ for particles with radius $a=2.0 \mu \mathrm{m}$. Line: prediction for rotation-translation coupling in the creeping-flow limit [19] for experimentally determined spring constants $\kappa_{1,2}=$ $2.6 \times 10^{-7} \mathrm{Nm}^{-1}$ and $s_{1,2}=2.5 \times 10^{-19} \mathrm{Nm} \mathrm{rad}^{-2}$ for translational and rotational traps, respectively.

from the relatively small number of decay times (around 1400) sampled in the experiment.

The positive sign of $C_{y_{1} \theta_{2}}(\tau)$ can be understood as follows. The cross-correlation function $C_{y_{1} \theta_{2}}(\tau)$ is a linear superposition of correlation functions of the four eigenmodes involving both relaxations of the transverse position fluctuations $\Delta y_{1,2}$ and of the angular fluctuations $\Delta \theta_{1,2}$ (see Fig. 4 in [19]). The behavior of $C_{y_{1} \theta_{2}}(\tau)$ at long times is governed by the slowest of these modes which is the one with $\Delta y_{1}>0$ and $\Delta \theta_{2}>0$ since in this mode the velocity fields excited by the position and orientation relaxations counteract each other, i.e., lead to enhanced friction. Thus for long times the cross-correlation function $C_{y_{1} \theta_{2}}(\tau)$ is positive. The peak in $C_{y_{1} \theta_{2}}(\tau)$ at short times arises from the superposition of 4 eigenmode correlation functions with equal amplitude, but opposite sign and slightly different relaxation times [19]. At $\tau=0$, the rotation-translation cross-correlation function vanishes, which reflects the absence of static angle-position correlations between the particles [10]. In our analysis we have assumed that the particles rotate only about the $z$ axis, and not about the $x$ and $y$ axes. In fact, the latter rotations are virtually unhindered, resulting in angular excursions that are, however, insignificant within the relaxation time of $C_{y_{1} \theta_{2}}(\tau)$ [19].

In summary, we have presented a direct measurement of the correlations between orientation and position fluctuations in colloidal suspensions mediated by hydrodynamic interactions, using direct imaging of birefringent particles trapped in both position and orientation by linearly polar- ized optical tweezers. While our measurements quantitatively confirm the theoretical predictions in the simple situation of two spheres in a fluid at low Reynolds number, the technique presented here could be useful for studying many-body correlations mediated by hydrodynamic interactions in more complex and possibly active systems, such as microfluidic devices or coupled beating cilia.

This work was funded by the Deutsche Forschungsgemeinschaft. S. Martin would like to acknowledge K. Mangold for experimental advice, and T. Gisler thanks R. Klein and G. Nägele for helpful discussions.

*Present address: Australian National University, Research School of Chemistry, Canberra, ACT 0200, Australia.

${ }^{\dagger}$ Present address: Max-Planck-Institut für Dynamik und Selbstorganisation, Bunsenstrasse 10, 37037 Göttingen, Germany.

${ }^{\ddagger}$ Electronic address: thomas.gisler@uni-konstanz.de

[1] M. Watzlawek and G. Nägele, J. Colloid Interface Sci. 214, 170 (1999).

[2] P. N. Segrè, E. Herbolzheimer, and P. M. Chaikin, Phys. Rev. Lett. 79, 2574 (1997).

[3] H. C. Berg, Phys. Today 53, No. 01, 24 (2000).

[4] M. Reichert and H. Stark, Eur. Phys. J. E 17, 493 (2005).

[5] A. Vilfan and F. Jülicher, Phys. Rev. Lett. 96, 058102 (2006).

[6] I. H. Riedel, K. Kruse, and J. Howard, Science 309, 300 (2005).

[7] S. Kim and S.-J. Karrila, Microhydrodynamics (Butterworth-Heinemann, Boston, 1991).

[8] J. Happel and H. Brenner, Low Reynolds Number Hydrodynamics (Noordhoff, Groningen, 1973).

[9] J.-C. Meiners and S. R. Quake, Phys. Rev. Lett. 82, 2211 (1999).

[10] P. Bartlett, S. I. Henderson, and S. J. Mitchell, Phil. Trans. R. Soc. Lond. A 359, 883 (2001).

[11] V. Degiorgio, R. Piazza, and R. B. Jones, Phys. Rev. E 52, 2707 (1995).

[12] R. B. Jones, Physica (Amsterdam) A150, 339 (1988).

[13] D. R. Cairns, N. S. Eichenlaub, and G. P. Crawford, Mol. Cryst. Liq. Cryst. 352, 275 (2000).

[14] K. Sandomirski, S. Martin, G. Maret, H. Stark, and T. Gisler, J. Phys. Condens. Matter 16, S4137 (2004).

[15] E. Fällman and O. Axner, Appl. Opt. 36, 2107 (1997).

[16] J. C. Crocker and D. G. Grier, J. Colloid Interface Sci. 179, 298 (1996); J. C. Crocker and E. R. Weeks, http:// www.physics.emory.edu/ weeks/idl.

[17] CRC Handbook of Chemistry and Physics, edited by D. R. Lide (CRC Press, Boca Raton, 1999), 80th ed.

[18] In fact, the exact form of $C_{y_{i} y_{i}}(\tau)$, being the sum of 4 exponentials with nearly equal decay times, is indistinguishable from a single exponential for the parameters used here [19].

[19] M. Reichert and H. Stark, Phys. Rev. E 69, 031407 (2004). 